\title{
Emerging Ultrastrong Coupling Between Light and Matter Observed in Circuit Quantum Electrodynamics
}

\author{
Kouichi Semba
}

\begin{abstract}
The strength of the coupling between an atom and a single electromagnetic field mode is defined as the ratio of the vacuum Rabi frequency to the Larmor frequency, and is determined by a small dimensionless physical constant, the fine structure constant $\alpha=Z_{v a c} / 2 R_{K}$. On the other hand, the quantum circuit including Josephson junctions behaving as artificial atoms and it can be coupled to the electromagnetic field with arbitrary strength (Devoret et al. 2007). Therefore, the circuit quantum electrodynamics (circuit QED) is extremely suitable for studying much stronger light-matter interaction.
\end{abstract}

We have used a Josephson junction atom, a flux qubit, harmonic oscillator coupled system. This circuit is well described by the Hamiltonian shown in Eq. (1).

$$
\mathcal{H}_{\text {total }}=-\frac{\hbar}{2}\left(\Delta \sigma_{x}+\varepsilon \sigma_{z}\right)+\hbar \omega_{\mathrm{o}}\left(\hat{a}^{\dagger} \hat{a}+\frac{1}{2}\right)+\hbar g \sigma_{z}\left(\hat{a}+\hat{a}^{\dagger}\right)
$$

The first, second, and third terms represent the energy of the qubit, the energy of the harmonic oscillator, and the interaction energy, respectively. If the coupling strength $\mathrm{g}$ becomes as large as the atomic and cavity frequencies ( $\Delta$ and $\omega_{o}$, respectively), the energy eigenstates including the ground state are predicted to be highly entangled (Hepp and Lieb 1973; Ashhab and Nori 2010). We have experimentally achieved this deep strong coupling using a superconducting-flux-qubit LC-oscillator system (Yoshihara et al. 2017). By carefully designing a superconducting persistent-current qubit interacting with an LC harmonic oscillator that has a large zero-point fluctuation current via a large shared Josephson inductance, we have realized circuits with $\frac{g}{\omega_{o}}$ ranging from 0.72 to 1.34 and $\frac{g}{\Delta} \gg 1$. From the transmission spectroscopy, we have observed unconventional transition spectra and selection rules which can be interpreted using predicted energy levels which are well described by Schrödingercat-like entangled states between persistent-current states and displaced vacuum or Fock states of the oscillator (Yoshihara et al. 2017). By using two-tone spectroscopy, the energies of the six lowest levels of each circuit have been determined. We have

\footnotetext{
K. Semba $(\varangle)$

National Institute of Information and Communications Technology, 4-2-1 Nukui-Kitamachi, Koganei, Tokyo 184-8795, Japan

e-mail: semba@nict.go.jp

(C) The Author(s) 2021

T. Takagi et al. (eds.), International Symposium on Mathematics, Quantum Theory, and Cryptography, Mathematics for Industry 33, https://doi.org/10.1007/978-981-15-5191-8_3
} 
observed huge light shifts, i.e., Lamb shifts, qubit energy shift due to coupling to vacuum field, that exceed $90 \%$ of the bare qubit frequencies and Stark shifts, inversions of the qubits' ground and excited states when there are only a few photons in the oscillator (Yoshihara et al. 2018). We have also observed collective coupling between an engineered 4300 ensemble of flux qubits and a superconducting resonator (Kakuyanagi et al. 2016), and considered the condition for observing generation of superradiant ground state in the presence of parameter fluctuations (Ashhab and Semba 2017).

\section{References}

S. Ashhab, F. Nori, Phys. Rev. A 81, 042311 (2010)

S. Ashhab, K. Semba, Phys. Rev. A 95, 053833 (2017)

M. Devoret, S. Girvin, R. Schoelkopf, Ann. Phys. (Leipzig) 16(10-11), 767-779 (2007)

K. Hepp, E.H. Lieb, Ann. Phys. (NY) 76, 360-404 (1973)

K. Kakuyanagi, Y. Matsuzaki, C. Deprez, H. Toida, K. Semba, H. Yamaguchi, W.J. Munro, S. Saito, Phys. Rev. Lett. 117, 210503 (2016)

F. Yoshihara, T. Fuse, S. Ashhab, K. Kakuyanagi, S. Saito, K. Semba, Nat. Phys. 13, 44 (2017)

F. Yoshihara, T. Fuse, Z. Ao, S. Ashhab, K. Kakuyanagi, S. Saito, T. Aoki, K. Koshino, K. Semba, Phys. Rev. Lett. 120, 183601 (2018)

Open Access This chapter is licensed under the terms of the Creative Commons Attribution 4.0 International License (http://creativecommons.org/licenses/by/4.0/), which permits use, sharing, adaptation, distribution and reproduction in any medium or format, as long as you give appropriate credit to the original author(s) and the source, provide a link to the Creative Commons license and indicate if changes were made.

The images or other third party material in this chapter are included in the chapter's Creative Commons license, unless indicated otherwise in a credit line to the material. If material is not included in the chapter's Creative Commons license and your intended use is not permitted by statutory regulation or exceeds the permitted use, you will need to obtain permission directly from the copyright holder. 